\title{
Qualitätsdimensionen für interaktive Systeme
}

\section{Stand der Arbeiten - UPA AK Qualitätsstandards}

\author{
Holger Fischer \\ s-lab I Software Quality Lab, Universität Paderborn \\ Zukunftsmeile 1 \\ 33102 Paderborn \\ holger.fischer@upb.de \\ Thomas Geis \\ ProContext Consulting $\mathrm{GmbH}$ \\ Unter Käster 14-16 \\ 50667 Köln \\ thomas.geis@procontext.de
}

\section{Kay Behrenbruch}

HFPS Humanergonomics UG

Dorfstraße 9

24259 Westensee

behrenbruch@humanergonomics.de

\author{
Knut Polkehn \\ artop $\mathrm{GmbH}$ \\ Christburger Straße 4 \\ 10405 Berlin \\ polkehn@artop.de \\ Oliver Kluge \\ Versicherungskammer Bayern \\ Maximilianstraße 53 \\ 80538 München \\ oliver.kluge@vkb.de \\ Catharina Riedemann \\ Olympus Soft Imaging Solutions $\mathrm{GmbH}$ \\ Johann-Krane-Weg 39 \\ 48149 Münster \\ catharina.riedemann@web.de
}

\begin{abstract}
Produktqualität ist gekennzeichnet durch das Ausmaß, in dem ein System die zuvor festgelegten Anforderungen erfüllt sowie die impliziten Erfordernisse der diversen Stakeholder befriedigt. Um eine gute Qualität zu erreichen, bedarf es eines erfolgreichen Qualitätsmanagements auf der Grundlage eines angemessenen Qualitätsmodells. Beim Thema Qualität liegt der Fokus häufig einseitig auf den Technologien (u.a. Wartbarkeit, Interoperabilität) und weniger auf den Menschen und den Geschäftszielen. Mit der Norm ISO/IEC 25010 - „System and software quality models“ - existiert ein internationaler Standard, der neben der technischen Perspektive sowohl die Qualitätscharakteristik Usability als auch Aspekte von „Qualität in der Nutzung“ (u.a. Effektivität, Effizienz) thematisiert. Allerdings existieren hierbei ungenutzte Potenziale sowie Widersprüche zu anderen Standards. Der Arbeitskreis arbeitet daher an normierbaren Qualitätsmerkmalen für ein konsistentes Qualitätsmodell.
\end{abstract}

\section{Keywords}

Qualitätsdimensionen, Messkriterien, Integration, Usability, User Experience 


\section{Einleitung}

In der heutigen Produktentwicklung, insbesondere von Softwareanwendungen, sind die Gebrauchstauglichkeit (Usability) sowie das Benutzererlebnis (User Experience, UX) als Qualitätscharakteristika seitens der softwareentwickelnden Unternehmen erkannt worden. Zudem werden sie häufig ebenfalls von den softwareanwendenden Unternehmen und Einzelanwendern explizit nachgefragt. Jedoch bleiben zahlreiche Softwareanwendungen weiterhin hinter ihren Potenzialen in Bezug auf diese Qualitätsaspekte zurück. Der Historie sowie der Interdisziplinarität der Softwareentwicklung geschuldet, wird der Begriff Usability - damit einhergehend auch der Begriff UX - von unterschiedlichen Personen unterschiedlich verstanden. Dabei wird er, aus den frühen Tagen der Mensch-Computer Interaktion entstehend, häufig lediglich als „,benutzerfreundlich“ oder „Einfachheit in der Nutzung“ übersetzt und somit ausschließlich auf die Benutzungsschnittstelle „über der Haube“ bezogen. Verstärkt wurde dies gegebenenfalls unter anderem dadurch, dass die Nützlichkeit und Funktionalität von der Usability abgegrenzt wurden (Nielsen 1994). Dies resultiert in der Praxis häufig in einer reinen „Oberflächenmaniküre“ - eine Softwareanwendung wird technologiegesteuert entwickelt und im Nachhinein noch grafisch aufbereitet (Seffah et al. 2005). Wesentliche Qualitätsaspekte, wie eine Angemessenheit in Bezug auf die Aufgaben der Benutzer oder eine Konformität in Hinblick auf deren Erwartungen, werden dabei vernachlässigt. Neben den offensichtlichen Auswirkungen der Gebrauchstauglichkeit hinsichtlich einer gesteigerten Effektivität, Effizienz und Zufriedenstellung bei den Benutzern bezogen auf die Erfüllung ihrer täglichen Aufgaben, handelt es sich beim HumanCentered Design ebenfalls um ein entscheidendes Attribut des Entwicklungsprozesses selbst (Fischer et al. 2011). Ein systematischer menschzentrierter Gestaltungsansatz ist daher notwendig, um interaktive Systeme mit einer vorhersagbaren Gebrauchstauglichkeit zu entwickeln. Zudem ist die Entwicklung moderner Softwaresysteme heutzutage durch einen hohen Zeitdruck mit kontinuierlich wechselnden Anforderungen geprägt, welcher durch eine Vielfalt technischer Fragestellungen und kürzer werdender Innovationszyklen zunehmend verstärkt wird (Biskup 2009). Somit entsteht insbesondere die Herausforderung einer Integration von Usability Engineering Aktivitäten in agile Softwareentwicklungsprozesse. Auch wenn dieses Thema in den letzten Jahren häufig behandelt wurde (Gothelf \& Seiden 2013; Kuusinen 2014), bleiben einige Herausforderungen im Hinblick auf die Synchronisierung der Taktung von Prozessen bestehen. So zeigt die Praxis, dass sich manche Formate wie Usability Testing, leichter in agilen Prozessen einbringen lassen als andere, wie bspw. Kontextanalysen oder Modellbildungen (Löffler et al. 2013).

Eine Ursache für häufig nicht erfolgreiche bzw. nur einen Teil der Anforderungen erfüllende Softwareprodukte liegt möglicherweise in der einseitigen Fokussierung auf technische Qualitätskriterien (z.B. Funktionalität, Zuverlässigkeit, Wartbarkeit, etc.). „Aus Sicht der Organisation bestimmt sich der Erfolg eines Produktes [jedoch] durch die Güte des Einklangs von Erfolgskriterien aus der Sicht des Business, der Benutzer und der Technologie“ (Löffler et al. 2013) (siehe Abbildung 1). 


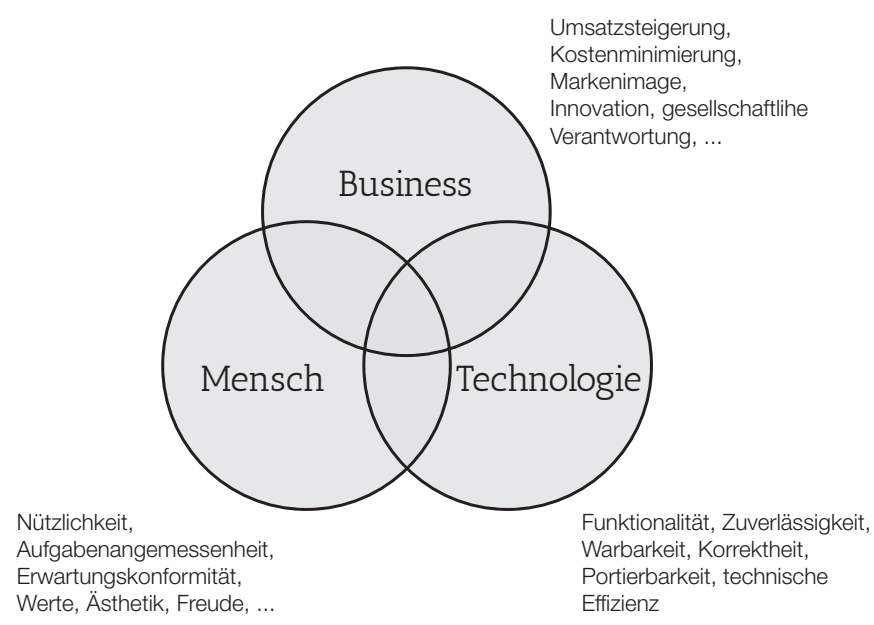

Abbildung 1: Qualitätsperspektiven für erfolgreiche Produkte (adaptiert nach Löffler et al. 2013)

Für eine erfolgreiche Integration von Usability und UX wäre demnach notwendig, vor der Ausgestaltung des Entwicklungsprozesses entsprechende Qualitätsziele für das Produkt zu definieren, so dass der Prozess auf diese ausgerichtet wird und entsprechende Techniken zur Erreichung der Qualitätsziele verortet sowie kommuniziert werden. Um angemessene Qualitätsmaße (Metriken) für ein Produkt im Vorfeld festlegen zu können, bedarf es allerdings eines geeigneten Qualitätsmodells, anhand dessen diese ausgewählt und gewichtet werden können. Somit können gleichzeitig Metriken festgelegt werden, die nach der Fertigstellung des Produktes entsprechend gemessen sowie für Softwaretests als auch Usability-Evaluationen herangezogen werden können. Solche Qualitätsmodelle existieren mittlerweile sowohl in Ansätzen (Seffah et al. 2006) als auch in normierter Form (ISO/IEC 25010), jedoch sind diese bislang $\mathrm{zu}$ sehr aus nur einer der oben genannten Perspektive formuliert.

Im Rahmen dieses Beitrags wird daher ein Konzept auf Basis existierender Ansätze vorgestellt, welches aktuell im Arbeitskreis Qualitätsstandards der German UPA ausgearbeitet wird. Dazu werden zunächst der Begriff Usability (auch als Grundlage für UX) erneut betrachtet sowie Qualitätsmodelle thematisiert, bevor der bisherige Stand sowie die anstehenden Arbeiten am Qualitätsmodell des Arbeitskreises präsentiert werden.

\section{Die Qualitätscharakteristik Usability}

Usability wird als Qualitätscharakteristik je nach Quelle und Domäne (bspw. Design, Softwareentwicklung, Qualitätsmanagement etc.) unterschiedlich definiert. Auch wenn in Kreisen von Usability Professionals überwiegend ,das Ausmaß, in dem ein Produkt durch bestimmte Benutzer in einem bestimmten Nutzungskontext genutzt werden kann, um 
bestimmte Ziele effektiv, effizient und zufriedenstellend zu erreichen" (ISO 9241-11) als Definition verwendet wird, so existieren dennoch ältere Definitionen anderer Domänen, insbesondere der Softwareentwicklung.

- „The ease with which a user can learn to operate, prepare inputs for, and interpret outputs of a system or component" (IEEE Std. 610.12-1990)

- „A set of attributes that bear on the effort needed for use and on the individual assessment of such use, by a stated or implied set of users“" (ISO/IEC 9126)

Diese werden derzeit teilweise durch die ISO 9241-11 adaptiert, wie es beim Übergang der ISO/IEC 9126 zur ISO/IEC 25010 der Fall war, jedoch sind sie bei den Vertretern der jeweiligen Domäne noch in den Köpfen verankert. Weitere in der Literatur bestehende Ansätze (e.g. Shackel 1991; Schneiderman 1992) liefern spezifischere Attribute zur Definition von Usability. Auch wenn diese im Kern Gemeinsamkeiten aufweisen (vgl. Tabelle 1), variieren sie in ihren Attributen. Neben der dadurch entstehenden allgemeinen Verwirrung von Forschern und Praktikern durch entstehende Kontroversen, fehlt weiterhin die entsprechende Tiefe zur Ableitung von Messkriterien. Ein angemessenes und konsensfähiges Qualitätsmodell, welches neben Usability auch die User Experience sowie die beiden weiteren Perspektiven (Business und Technologie) berücksichtigt, könnte diese Kontroversen bereinigen und eine Grundlage für Messkriterien darstellen.

\begin{tabular}{|c|c|c|c|c|c|}
\hline $\begin{array}{l}\text { ISO 9241-11 } \\
(1998)\end{array}$ & $\begin{array}{l}\text { Constantine \& } \\
\text { Lockwood } \\
(1999)\end{array}$ & $\begin{array}{l}\text { Preece et al. } \\
\text { (1994) }\end{array}$ & Nielsen (1993) & $\begin{array}{l}\text { Shneiderman } \\
\text { (1992) }\end{array}$ & $\begin{array}{l}\text { Shackel } \\
(1991)\end{array}$ \\
\hline \multirow[t]{4}{*}{ Efficiency } & $\begin{array}{l}\text { Efficiency in } \\
\text { use }\end{array}$ & Throughput & $\begin{array}{l}\text { Efficiency of } \\
\text { use }\end{array}$ & $\begin{array}{l}\text { Speed of } \\
\text { performance }\end{array}$ & $\begin{array}{l}\text { Effectiveness } \\
\text { (Speed) }\end{array}$ \\
\hline & Learnability & $\begin{array}{l}\text { Lernability } \\
\text { (Ease of } \\
\text { learning) }\end{array}$ & $\begin{array}{l}\text { Learnability } \\
\text { (Ease of } \\
\text { learning) }\end{array}$ & Time to learn & $\begin{array}{l}\text { Learnability } \\
\text { (Time to learn) }\end{array}$ \\
\hline & $\begin{array}{l}\text { Remember- } \\
\text { ability }\end{array}$ & & Memorability & $\begin{array}{l}\text { Retention over } \\
\text { time }\end{array}$ & $\begin{array}{l}\text { Learnability } \\
\text { (Retention) }\end{array}$ \\
\hline & $\begin{array}{l}\text { Reliability in } \\
\text { use }\end{array}$ & Throughput & Errors/safety & $\begin{array}{l}\text { Rate of errors } \\
\text { by users }\end{array}$ & $\begin{array}{l}\text { Effectiveness } \\
\text { (Errors) }\end{array}$ \\
\hline $\begin{array}{l}\text { Satisfaction } \\
\text { (Comfort and } \\
\text { acceptability of } \\
\text { use) }\end{array}$ & $\begin{array}{l}\text { User } \\
\text { satisfaction }\end{array}$ & Attitude & Satisfaction & $\begin{array}{l}\text { Subjective } \\
\text { satisfaction }\end{array}$ & Attitude \\
\hline
\end{tabular}

Tabelle 1: Attribute zur Verfeinerung der Definition von Usability (adaptiert nach Seffah et al. 2006) 


\section{Ein überarbeitetes Qualitätsmodell}

Mit der ISO/IEC 25010 „Systems and Software Engineering - Systems and Software Quality Requirements and Evaluation (SQuaRE) - System and Software Quality Models“ (2011) existiert ein normiertes Konzept für Qualitätsmodelle in der Softwareentwicklung. Da die Perspektive auf die Qualität einer Software hierbei vorrangig aus dem Software Engineering betrachtet wurde, wird die Usability im Wesentlichen als Qualität „über der Haube“ und somit ohne Abhängigkeit zur Funktionalität einer Software dargestellt. Ebenfalls bleibt die User Experience noch weitestgehend unberücksichtigt bzw. wird sie lediglich in Ansätzen unter dem Qualitätsaspekt „Quality in Use, Teilaspekt Satisfaction“ adressiert. Dennoch enthält die Norm ein grundlegendes Modell zur Strukturierung von Qualitätsaspekten, welches in seiner Ausprägung als angemessen erscheint. Dieses ist unterteilt in Qualitätscharakteristika, Qualitätssubcharakteristika sowie Qualitätsmerkmale. Während die Charakteristika und Subcharakteristika auf eine feingranulare Unterteilung der Qualitätsaspekte abzielen, wird durch ein Qualitätsmerkmal auf der untersten Ebene eine messbare Größe beschrieben.

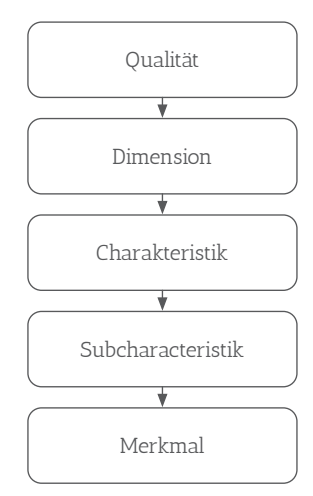

Abbildung 2: Ebenen des Qualitätsmodells (adaptiert nach ISO/IEC 25010)

Das Konzept aus der Norm wurde in diesem Ansatz entsprechend aufgegriffen und um die Ebene der Qualitätsdimension erweitert (vgl. Abbildung 2). Somit können die drei weiter oben beschriebenen Perspektiven „Business“, „Mensch“ und „Technologie“ eine jeweils gleichberechtigte Gewichtung erhalten. Die Auswahl und jeweilige Gewichtung der Qualitätsmerkmale bleibt jedoch abhängig vom jeweiligen Projekt und den darin agierenden Personen bzw. Rollen. Die drei Perspektiven wurden in die entsprechenden drei Dimensionen übertragen und für den internationalen Diskurs in Englisch formuliert: Business-Centered Quality, Human-Centered Quality, Technology-Centered Quality.

Die Zielgruppe dieses Ansatzes ist somit breit aufgestellt und enthält vor allem die entsprechenden Rollen im Projekt (bspw. Qualitätsmanagement, Produktmanagement), die für die Definition der qualitativen Ziele, die mit dem Produkt erreicht werden sollen, verantwortlich sind. 
Für eine erste Version eines Qualitätsmodells wurden aus der ISO/IEC 25010 die Aspekte der Produktqualität in die Dimension Technology-Centered Quality eingeordnet. Dabei wurde bewusst die Usability nicht berücksichtigt. Die Dimension Business-Centered Quality wird zunächst für eine spätere Ausarbeitung vorgesehen. Der Fokus der Arbeit liegt somit auf der Dimension Human-Centered Quality. In dieser Dimension wurden die Subcharakteristika der Usability grundlegend umstrukturiert und unter anderem um die Dialogprinzipien der ISO 9241-110 (2006) erweitert. Des Weiteren wurden neben der Usability einer Softwareanwendung die Charakteristika der User Experience sowie Accessibility hinzugefügt. Ein entsprechender Ausschnitt des aktuellen Standes am Qualitätsmodell ist in Abbildung 3 dargestellt.

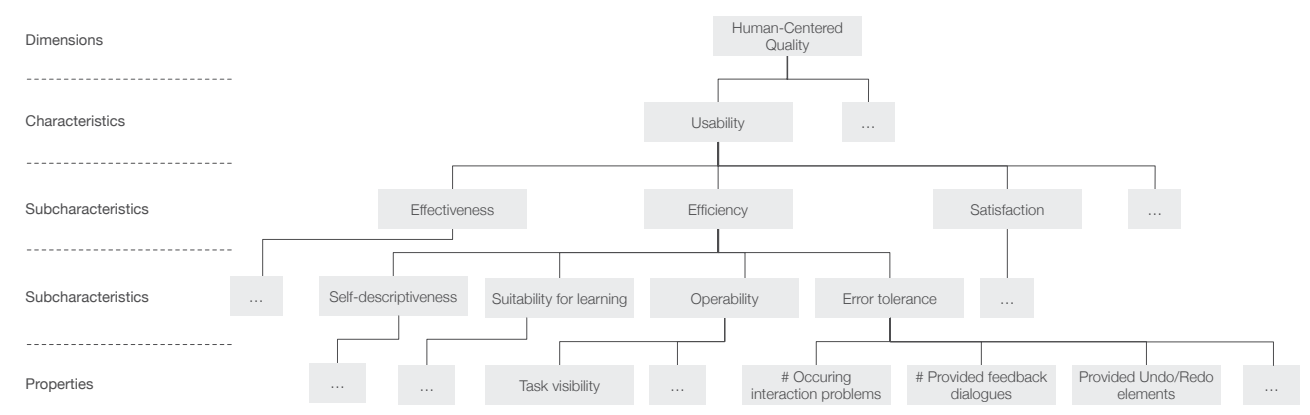

Abbildung 3: Ausschnitt der Human-Centered Quality des Qualitätsmodells

Eine weitere Verfeinerung der Dimension Human-Centered Quality lässt sich im Qualitätsmodell durch die Qualitätsmerkmale feststellen, da erst auf dieser Ebene messbare Kriterien entstehen, die bei der Bewertung eines Produktes herangezogen werden können. Die ISO/IEC 25010 definiert zwar bereits diese Ebene im generellen Qualitätsmodell, bleibt jedoch noch eine entsprechende Ausgestaltung im Produktqualitätsmodell schuldig. Ein Ansatz, welcher messbare Kriterien in Bezug auf die Usability eines Produktes formuliert, ist in Seffah et al. (2006) zu finden. Auch hier wird ein Qualitätsmodell formuliert. Der Fokus dieses Qualitätsmodell liegt ausschließlich auf der Usability eines Produktes und betrachtet weder die Charakteristiken User Experience und Accessibility, noch die Dimensionen Business und Technologie. Die messbaren Kriterien nach Seffah et al. (2006) in Bezug auf Usability wurden in dieser Arbeit gesichtet und werden sukzessive ins Qualitätsmodell überführt.

\section{Zusammenfassung \& Ausblick}

In diesem Beitrag wurde der Ansatz eines Qualitätsmodells adaptiert von existierenden Konzepten der ISO/IEC 25010 sowie Seffah et al. (2006) vorgestellt. Dieses wurde untergliedert in die drei Dimensionen Business, Mensch und Technologie. Somit soll herausgestellt werden, dass die Qualität und der Erfolg eines Produktes nicht nur von einer technischen Seite definiert werden. Basierend auf diesem Qualitätsmodell sollen 
Qualitätskriterien ausgewählt sowie gewichtet werden. Diese können sowohl einer erfolgreichen Integration von Usability und UX in die Softwareentwicklung zu Grunde gelegt werden als auch als Metriken dienen, die nach der Fertigstellung des Produktes entsprechend gemessen sowie für Softwaretests als auch Usability-Evaluationen herangezogen werden können.

Das Qualitätsmodell befindet sich momentan in der weiteren Ausarbeitung durch den Arbeitskreis Qualitätsstandards der German UPA. Eine bislang offene Fragestellung und somit Potenzial für weitere Arbeiten besteht in der Struktur des Qualitätsmodells. Bislang sind die Qualitätscharakteristika in Form einer Taxonomie angeordnet. Da sich Qualitätskriterien gegebenenfalls aber auch gegenseitig bedingen können, könnte beispielsweise eine Ontologie eine angemessenere Repräsentation darstellen, um Abhängigkeiten abzubilden.

\section{Literatur}

Biskup. T. (2009). Agile fachmodellgetriebene Softwareentwicklung für mittelständische IT-Projekte. Dissertation, Universität Oldenburg.

Dzida, W. (1994). Qualitätssicherung durch software-ergonomische Normen. In Eberleh, E. (Hrsg.): Einführung in die Software-Ergonomie. Berlin: de Gruyter-Verlag, S. 373-406.

Constantine, L.L. \& Lockwood, L.A.D. (1999). Software for Use: A Practical Guide to the Models and Methods of Usage-Centered Design. New York, USA: Addison-Wesley.

Fischer, H., Nebe. K. \& Klompmaker, F. (2011). A Holistic Model for Integrating Usability Engineering and Software Engineering Enriched with Marketing Activities. In Kurosu, M. (Hrsg.): Human Centered Design, HCII 2011, LNCS 6776. Berlin Heidelberg: Springer, S. 28-37.

Gothelf, J. \& Seiden, J. (2013). Lean UX-Applying Lean Principles to Improve User Experience. Sebastopol, CA, USA: O’Reilly Media, Inc.

IEEE Std. 610.12 (1990). IEEE Standard Glossary of Software Engineering Terminology. IEEE Computer Society, Washington, DC, USA.

ISO/IEC 9126-1 (2001). Software Engineering - Product quality - Part 1: Quality model. International Organization for Standardization (ISO), Vernier, Schweiz.

ISO 9241-11 (1998). Ergonomic requirements for office work with visual display terminals (VDTs) Part 11: Guidance on usability. International Organization for Standardization (ISO), Vernier, Schweiz.

ISO 9241-110 (2006). Ergonomic of human-system interaction - Part 110: Dialogue principles. International Organization for Standardization (ISO), Vernier, Schweiz.

ISO/IEC 25010 (2011). Systems and software Engineering - Systems and software Quality Requirements and Evaluation (SQuaRE) - System and software quality models. International Organization for Standardization (ISO), Vernier, Schweiz.

Kuusinen, K. (2014). Improving UX Work in Scrum Development: A Three-Year Follow-Up Study in a Company. In Sauer, S. et al. (Hrsg.): Proceedings of the 5th International Conference on HumanCentered Software Engineering, HCSE 2014, LNCS 8742. Heidelberg: Springer, S. 259-266. 
Löffler, D., Polkehn, K. \& Hüttner, J. (2013). Erfolgreiche Usability \& UX in Unternehmen - Thesen und Erfolgsfaktoren zu Usability/UX-Prozessen, -Strategien und Change. In Brau, H., Lehmann, A., Petrovic, K. \& Schröder, M.C. (Hrsg.): Tagungsband UP13. Stuttgart: German UPA e.V., S. $22-25$.

Nielsen, J. (1994). Usability Engineering. London, Großbritannien: Academic Press.

Preece, J., Rogers, Y. \& Sharp, H. (1994). Human Computer Interaction-Concepts and Design. Wokingham, Großbritannien: Addison-Wesley.

Shneiderman, B. (1992). Designing the User Interface: Strategies for Effective Human-Computer Interaction, 2nd edition. Reading, MA, USA: Addison-Wesley.

Seffah, A., Gulliksen, J. \& Desmarais, M.C. (2005). An Introduction to Human-Centered Software Engineering: Integrating Usability in the Development Process. In Seffah, A., Gulliksen, J. \& Desmarais, M.C. (Hrsg.): Human-Centered Software Engineering - Integrating Usability in the Software Development Lifecycle. Dordrecht, NL: Springer, S. 3-14.

Seffah, A., Donyaee, M., Kline, R.B. \& Padda, H.K. (2006). Usability measurement and metrics: A consolidated model. Software Quality Journal (14). Berlin: Springer Science + Business Media, Inc., S. 159-178.

Shackel, B. (1991). Usability - Context, framework, definition, design and evaluation. In Shakel, B. \& Richardson, S. (Hrsg.): Human Factors for Informatics Usability. Cambridge, MA, USA:

University Press, S. 21-38. 